\title{
Static Balance in Patients with Vestibular Impairments: A Preliminary Study
}

\author{
Hossein Talebi, ${ }^{1,2}$ Mohammad Taghi Karimi, ${ }^{3}$ \\ Seyed Hamid Reza Abtahi, ${ }^{4}$ and Niloofar Fereshtenejad ${ }^{3}$ \\ ${ }^{1}$ Audiology Department, Musculoskeletal Research Center, Faculty of Rehabilitation, Isfahan University of Medical Sciences, \\ Isfahan 81746-73461, Iran \\ ${ }^{2}$ Communication Disorders Research Center, Faculty of Rehabilitation, Isfahan University of Medical Sciences, \\ Isfahan 81746-73461, Iran \\ ${ }^{3}$ Musculoskeletal Research Center, Faculty of Rehabilitation, Isfahan University of Medical Sciences, Isfahan 81746-73461, Iran \\ ${ }^{4}$ Faculty of Medicine, Isfahan University of Medical Sciences, Isfahan 81746-73461, Iran
}

Correspondence should be addressed to Hossein Talebi; ht6023@gmail.com

Received 20 December 2015; Revised 20 April 2016; Accepted 17 May 2016

Academic Editor: Bijan Najafi

Copyright (C) 2016 Hossein Talebi et al. This is an open access article distributed under the Creative Commons Attribution License, which permits unrestricted use, distribution, and reproduction in any medium, provided the original work is properly cited.

\begin{abstract}
Aims. Vestibular system is indicated as one of the most important sensors responsible for static and dynamic postural control. In this study, we evaluated static balance in patients with unilateral vestibular impairments. Materials and Methods. We compared static balance control using Kistler force plate platform between 10 patients with unilateral vestibular impairments and 20 normal counterparts in the same sex ratio and age limits $(50 \pm 7)$. We evaluated excursion and velocity of center of pressure (COP) and path length in anteroposterior (AP) and mediolateral (ML) planes with eyes open and with eyes closed. Results. There was no significant difference between COP excursions in ML and AP planes between both groups with eyes open and eyes closed ( $p$ value $>0.05)$. In contrast, the difference between velocity and path length of COP in the mentioned planes was significant between both groups with eyes open and eyes closed ( $p$ value $<0.05$ ). Conclusions. The present study showed the static instability and balance of patients with vestibular impairments indicated by the abnormal characteristics of body balance.
\end{abstract}

\section{Introduction}

Postural control is the ability to control body or posture automatically or in response to external changes [1]. Vestibular, visual, and somatosensory systems are essential and responsible for the control of posture [2]. Afferents of these systems are processed and integrated in multiple structures of central nervous system. Then, motor commands are sent to musculoskeletal system [2]. It is indicated that postural control system plays an important role in maintenance of balance on the small support base provided by the feet [3].

Vestibular system plays an important role in control of static and dynamic posture and balance. Any impairments of movement perception, vertical orientation, control of center of body position, and head fixation result in gait and balance problems [3]. Vestibular pathologies result in balance impairments in 50\% of cases [3]. In these conditions, dizziness is one of the most common complaints reported by the patients [3]. Therefore, comprehensive evaluation of the patients with balance disorders is very important.

Instability and postural imbalance are commonly demonstrated as body sway increase in situations with impaired coordination between visual and proprioceptive systems, decrease in threshold of stability and functional capacity, gait changes, and falling in patients with vestibular impairments. Falling (the primary consequence of postural imbalance) is associated with various impairments in neuromuscular system. This condition is resulting from an inability to maintain normal posture [4]. There are some tests that evaluated standing balance in static and dynamic conditions. Berg Balance Scale was a subjective test designed to evaluate standing balance in elderly patients [5]. This scale predicts falling in 
seniors and is sensitive to change after vestibular rehabilitation [6]. The Get Up and Go Test designed to identify elderly fallers is a test of walking balance. It differentiates elderly patients with high risk of falling from those patients with low risk of falling [7]. Vestibuloocular reflex (VOR) and vestibulospinal reflex (VSR) are considered for the objective assessment of postural stability in patients with vestibular impairments [4]. VOR is indicated as a system of primary control of visual stabilization during locomotion and any disturbances of VOR result in dizziness and other symptoms of body imbalance [8]. Assessment of VOR and VSR is essential for vestibular, visual, and somatosensory systems responsible for balance control [9]. Many studies highlighted more detailed information about the role of VOR in patients with vestibular impairments. In contrast, far more studies are required for assessment of VSR and proprioception in the mentioned patients.

In the present study, we evaluated this reflex and the proprioception in more detail using force plate platform in patients with vestibular impairments. Our primary hypothesis was that this reflex could be affected in the patients with vestibular impairments. The results of this study surely shed light on and increase our knowledge about the effects of vestibular impairments on VSR and proprioception and better designing of balance rehabilitation programs.

It is indicated that the assessment of VSR as well as proprioception has been conducted in patients with vestibular impairments mostly with posturography [10]. In the present study, we measured many components of static balance such as center of pressure (COP) in anteroposterior (AP) and mediolateral (ML) directions, path length (PL), and velocity in the mentioned directions using force plate platform.

\section{Materials and Methods}

10 patients with unilateral vestibular impairments and 20

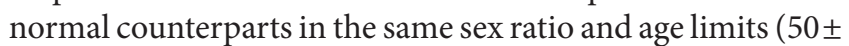
7) participated in this study. The patients were referred from ear, nose, and throat clinic of Alzahra Hospital of Isfahan University of Medical Sciences. All of the patients showed evidence of vestibular impairments such as true vertigo. These patients were evaluated by an otolaryngologist and an expert audiologist using videonystagmography (VNG) test. All of the patients had at least $20 \%$ weakness on bithermal caloric testing in the left ear. The patients also had decreased vestibuloocular reflex responses to low frequency sinusoidal rotations in darkness. All of the patients have been medicated by ENT surgeons.

2.1. Inclusion Criteria. Inclusion criteria were as follows: presence of vestibular impairments, age limit between 30 and 60 years, no evidence of cerebellar ataxia, no evidence of effusions in the middle ear, normal condition in musculoskeletal system and normal standing and gait, normal visual function, and no evidence of neurologic disorders such as multiple sclerosis and sensory impairments of the lower limbs in the diabetic patients.
2.2. Exclusion Criteria. Exclusion criteria were as follows: all of the patients with Meniere's disease, migraine, joint replacements, history of neurologic disease, and functional vision with their corrective lenses had been excluded from this study.

The present study was in accordance with Helsinki Declaration. We conducted this project in Musculoskeletal Research Center of Isfahan University of Medical Sciences. An ethical approval was obtained from ethical committee of Isfahan University of Medical Sciences (Ethics Committee reference number IR.MUI.REC.1394.2.094). Moreover, every subject was asked to sign a consent form before data collection. The static balance of the participants while standing was evaluated by use of Kistler force plate (Kistler Instrument Corp., NY, United States) when they were standing for one minute with eyes open and eyes closed. They were asked to stand on force plate for one minute in a comfortable position. The subjects were asked to keep their eyes either open or closed while maintaining the stance (feet together and arms to the sides) for $60 \mathrm{~s}$ [11]. The data were collected with frequency of $120 \mathrm{~Hz}$ and were filtered with Butterworth low pass filter with cutoff frequency of $10 \mathrm{~Hz}$. The first and last 15 seconds of the data were deleted to remove the effects of sudden standing on the force plate and also the effects of fatigue, respectively. The tests were repeated to collect 5 trials for each condition (eyes open and eyes closed). It should be emphasized that the selection of conditions to be tested was done randomly. The stability parameters for static balance of the participants were evaluated by use of the following equations:

$$
\begin{aligned}
\operatorname{COPEAP}(\mathrm{mm}) & =X_{\max }-X_{\text {min }}, \\
\operatorname{COPEML}(\mathrm{mm}) & =Y_{\max }-Y_{\text {min }}, \\
\operatorname{PLAP}(\mathrm{mm}) & =\sum \sqrt[n-1]{\left(x_{i+1}-x_{i}\right)^{2}}, \\
\operatorname{PLML}(\mathrm{mm}) & =\sum \sqrt[n-1]{\left(y_{i+1}-y_{i}\right)^{2}}, \\
\operatorname{VAP}(\mathrm{mm} / \mathrm{min}) & =\frac{\sum^{n-1} \sqrt{\left(x_{i+1}-x_{i}\right)^{2}}}{t}, \\
\operatorname{VML}(\mathrm{mm} / \mathrm{min}) & =\frac{\sum^{n-1} \sqrt{\left(y_{i+1}-y_{i}\right)^{2}}}{t},
\end{aligned}
$$

where $X$ plane is the displacement in the anteroposterior direction and $Y$ plane is the displacement in the mediolateral direction. In addition, COPEAP, COPEML, PLAP, PLML, VAP, and VML are the excursion of the center of pressure in the anteroposterior direction, excursion of the center of pressure in the mediolateral direction, path length in the anteroposterior direction, path length in the mediolateral direction, velocity of the COP in the anteroposterior direction, and velocity of the COP in the mediolateral direction, respectively.

2.3. Statistical Analysis. We measured the mean of the parameters for 5 test trials. In addition, we compared static balance 
TABLE 1: The characteristics (mean \pm SD) of the subjects that participated in this study.

\begin{tabular}{lcccccc}
\hline Participants & Number & Age & Mass & Height $(\mathrm{m})$ & BMI $\left(\mathrm{kg} / \mathrm{m}^{2}\right)$ & Sex $($ female $)$ \\
\hline Patients & 10 & $50 \pm 7$ & $59 \pm 14$ & $1.7 \pm 0.25$ & $20.50 \pm 5.2$ & 5 \\
Normal & 20 & $50 \pm 5$ & $61 \pm 12.5$ & $1.7 \pm 0.15$ & $21.20 \pm 4.3$ & 10 \\
\hline
\end{tabular}

TABLE 2: The mean values with standard deviation of the stability parameters of normal subjects and the patients.

\begin{tabular}{cccccccc}
\hline \multicolumn{2}{c}{ Participants } & COPEAP $(\mathrm{mm})$ & COPEML $(\mathrm{mm})$ & VAP $(\mathrm{mm} / \mathrm{min})$ & VML $(\mathrm{mm} / \mathrm{min})$ & PLAP $(\mathrm{mm})$ & PLML $(\mathrm{mm})$ \\
\hline \multirow{4}{*}{ Eyes open } & Normal & $25.25 \pm 10.82$ & $13.76 \pm 5.32$ & $928.22 \pm 113.5$ & $1004.45 \pm 154.43$ & $460.77 \pm 62.62$ & $502.19 \pm 77.2$ \\
& Patients & $25.93 \pm 9.6$ & $18.18 \pm 7.04$ & $2688.83 \pm 1139.73$ & $1939.89 \pm 621.34$ & $1345.75 \pm 571.12$ & $970.62 \pm 310.35$ \\
& $p$ value & 0.43 & $0.06^{*}$ & $0.00^{*}$ & $0.00^{*}$ & $0.00^{*}$ & $0.00^{*}$ \\
\hline \multirow{3}{*}{ Eyes closed } & Normal & $24.21 \pm 9.76$ & $14.55 \pm 8.06$ & $985.56 \pm 160.49$ & $1005.38 \pm 173.27$ & $492.31 \pm 80.69$ & $502.88 \pm 86.68$ \\
& Patients & $30.55 \pm 18.56$ & $19.46 \pm 12.00$ & $2470 \pm 1023$ & $1974.33 \pm 698.81$ & $1235 \pm 511.5$ & $987.14 \pm 349.42$ \\
& $p$ value & 0.18 & 0.14 & $0.00^{*}$ & $0.001^{*}$ & $0.001^{*}$ & $0.001^{*}$ \\
\hline
\end{tabular}

${ }^{*}$ Significant difference between normal subjects and patients with vestibular impairments at $p<0.05$.

between patients with vestibular impairments and normal participants with two-sample $t$-test. The significant point at 0.05 was used for final analysis. The statistical test was done using SPSS software (version 21, Chicago, USA).

\section{Results}

Table 1 shows the characteristics of the subjects that participated in this study. The mean values of COPEAP and COPEML in the patients with eyes open were $25.93 \pm 9.6$ and $18.18 \pm 7.04 \mathrm{~mm}$, respectively, compared to $25.25 \pm 10.82$ and $13.76 \pm 5.32 \mathrm{~mm}$ for normal subjects (the difference between the mean values between both groups was not significant, $p$ value $>0.05$ ).

There was also no significant difference between COPEAP and COPEML between both groups with eyes closed ( $p$ value $>0.05)$. In contrast, the difference between VAP and VML between both groups with eyes open and eyes closed was significant ( $p$ value $=0.00$ ). The PLAP and PLML were $460.77 \pm 62.62$ and $502.19 \pm 77.2$ and $1345.75 \pm 571.12$ and $970.62 \pm 310.35 \mathrm{~mm}$, for normal cases and patients, respectively ( $p$ value $=0.00$ ). Also, with eyes closed, there was significant difference between normal participants and patients for PLAP and PLML ( $p$ value $=0.01$ ). Table 2 summarizes the stability parameters of both normal participants and the patients.

\section{Discussion}

There is no doubt that the risk of falling mostly depends on static and dynamic stability of subjects. Various neuromuscular disorders influence the stability of subjects while standing and walking [12-15]. In addition, some patients suffer from vestibular impairments which influence their abilities to manage their stability $[11,15,16]$. The present study compared the stability of the patients with vestibular impairments with that of normal subjects during quiet standing.

The results showed that although it seems that these patients have the same stability as that of normal subjects (based on COP excursions), they have impaired stability. They have the same excursion while standing as normal subjects but they have continued sways. It means that their sway range is the same as normal but they do not have a constant stability. Their COP moved from anterior to posterior position and from lateral to medial position simultaneously with high frequency. In other words, they cannot control the movement of COP. These results support findings reported in some researches that instability was consistently increased in patients with vestibular impairments $[11,15]$.

This study suggested that unilateral vestibular dysfunction increased postural instability compared to control participants. The patients showed a significant increase approximately in all parameters of static stability. Our results demonstrated that the parameters assessed by force plate platform could be sensitive in showing any alterations in static stability of patients with vestibular impairments. This study highlighted the static instability of patients with vestibular impairments indicated by the abnormal characteristics of body balance. Some studies showed that the kinetics provide a useful third level of analysis for patients with vestibular impairments $[17,18]$. These analyses with the other balance measurements including Berg Balance Scale (Berg), Dynamic Gait Index (DGI), Timed Up and Go (TUG), Computerized Dynamic Posturography Sensory Organization Test (SOT), and VNG are sensitive in identifying patients with vestibular impairments $[17,18]$. For this preliminary study, our data may not be strictly comparable to the general population. These data should be interpreted with caution, however, due to the decreased sample size. In addition, further research could be suggested to better identify the consequences of vestibular impairments on static and dynamic stability.

\section{Conclusion}

In this research, the measured parameters of static balance including center of pressure, path length, and velocity in anteroposterior and mediolateral directions show static balance abnormality in patients with vestibular impairments. 


\section{Competing Interests}

The authors declared no potential conflict of interests with respect to the research, authorship, and/or publication of this paper.

\section{Acknowledgments}

The authors are grateful to all the patients and normal subjects that participated in this study. This work was supported by the Musculoskeletal Research Center of Isfahan University of Medical Sciences (Grant no. 294094).

\section{References}

[1] R. K. Kenton, "Objective assessment of posture and gait," in Clinical Disorders of Balance, Posture and Gait, A. M. Bronstein, T. Brandt, M. H. Woollacott, and J. G. Nutt, Eds., pp. 130-146, Arnold, London, UK, 2nd edition, 2004.

[2] R. B. García, S. P. Corresa, J. M. B. Bertomeu, and M. M. M. Suárez-Varela, "Static posturography with dynamic tests. Usefulness of biomechanical parameters in assessing vestibular patients," Acta Otorrinolaringologica Espanola, vol. 63, no. 5, pp. 332-338, 2012.

[3] R. M. Quitschal, J. Y. Fukunaga, M. M. Ganança, and H. H. Caovilla, "Evaluation of postural control in unilateral vestibular hypofunction," Brazilian Journal of Otorhinolaryngology, vol. 80, no. 4, pp. 339-345, 2014.

[4] J. M. L. Katz, R. Burkard, and L. J. Hood, Handbook of Clinical Audiology, Lippincott Williams \& Wilkins, 2009.

[5] K. Berg, S. Wood-Dauphinee, and J. I. Williams, "The balance scale: reliability assessment with elderly residents and patients with an acute stroke," Scandinavian Journal of Rehabilitation Medicine, vol. 27, no. 1, pp. 27-36, 1995.

[6] S. R. Ados and J. S. Pereira, "Balance improvement and reduction of likelihood of falls in older women after Cawthorne and Cooksey exercises," Brazilian Journal of Otorhinolaryngology, vol. 71, no. 1, pp. 38-46, 2005.

[7] S. Mathias, U. S. L. Nayak, and B. Isaacs, "Balance in elderly patients: the 'get-up and go' test," Archives of Physical Medicine and Rehabilitation, vol. 67, no. 6, pp. 387-389, 1986.

[8] M. M. Gananca, H. H. Caovilla, M. S. L. Munhoz, M. L. G. Silva, F. F. Gananca, C. F. Gananca et al., "O que esperar da equilibriometria," in Equilibriometria Clínica, H. H. Caovilla, M. M. Gananca, M. S. L. Munhoz, and M. L. G. Silva, Eds., pp. 23-29, Atheneu, São Paulo, Brazil, 1999.

[9] F. O. Black and W. H. Paloski, "Computerized dynamic posturography: what have we learned from space?" OtolaryngologyHead and Neck Surgery, vol. 118, no. 3, pp. S45-S51, 1998.

[10] R. Kohen-Raz, A. Sokolov, A. Kohen-Raz, M. Demmer, and M. Harell, "Posturographic correlates of peripheral and central vestibular disorders as assessed by electronystagmography (ENG) and the Tetrax Interactive Balance System," in Intercranial and Inner Ear Physiology and Pathophysiology, A. Reid, R. Marchbanks, and A. Ernst, Eds., pp. 231-236, Whurr, London, UK, 1998

[11] M. Aoki, T. Tokita, B. Kuze, K. Mizuta, and Y. Ito, "A characteristic pattern in the postural sway of unilateral vestibular impaired patients," Gait \& Posture, vol. 40, no. 3, pp. 435-440, 2014.

[12] G. R. Cybulski and R. J. Jaeger, "Standing performance of persons with paraplegia," Archives of Physical Medicine and Rehabilitation, vol. 67, no. 2, pp. 103-108, 1986.
[13] T. E. Prieto, J. B. Myklebust, R. G. Hoffmann, E. G. Lovett, and B. M. Myklebust, "Measures of postural steadiness: differences between healthy young and elderly adults," IEEE Transactions on Biomedical Engineering, vol. 43, no. 9, pp. 956-966, 1996.

[14] J. Swanenburg, E. D. de Bruin, K. Favero, D. Uebelhart, and T. Mulder, "The reliability of postural balance measures in single and dual tasking in elderly fallers and non-fallers," $B M C$ Musculoskeletal Disorders, vol. 9, article 162, 2008.

[15] R. W. Baloh, K. M. Jacobson, K. Beykirch, and V. Honrubia, "Static and dynamic posturography in patients with vestibular and cerebellar lesions," Archives of Neurology, vol. 55, no. 5, pp. 649-654, 1998.

[16] J.-R. Yeh, M.-T. Lo, F.-L. Chang, and L.-C. Hsu, "Complexity of human postural control in subjects with unilateral peripheral vestibular hypofunction," Gait \& Posture, vol. 40, no. 4, pp. 581586, 2014.

[17] H. S. Cohen and K. T. Kimball, "Usefulness of some current balance tests for identifying individuals with disequilibrium due to vestibular impairments," Journal of Vestibular Research: Equilibrium and Orientation, vol. 18, no. 5-6, pp. 295-303, 2008.

[18] H. S. Cohen, A. P. Mulavara, B. T. Peters, H. Sangi-Haghpeykar, and J. J. Bloomberg, "Standing balance tests for screening people with vestibular impairments," Laryngoscope, vol. 124, no. 2, pp. 545-550, 2014. 


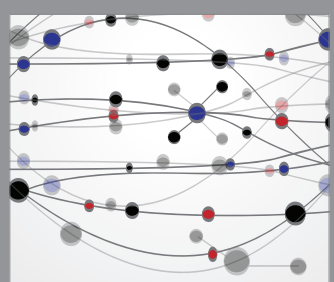

The Scientific World Journal
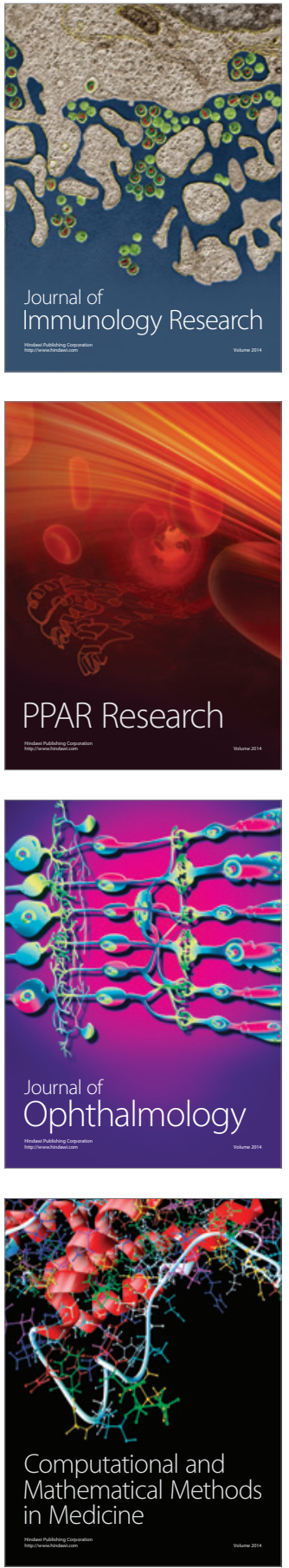

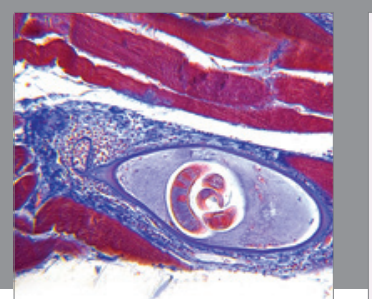

Gastroenterology Research and Practice

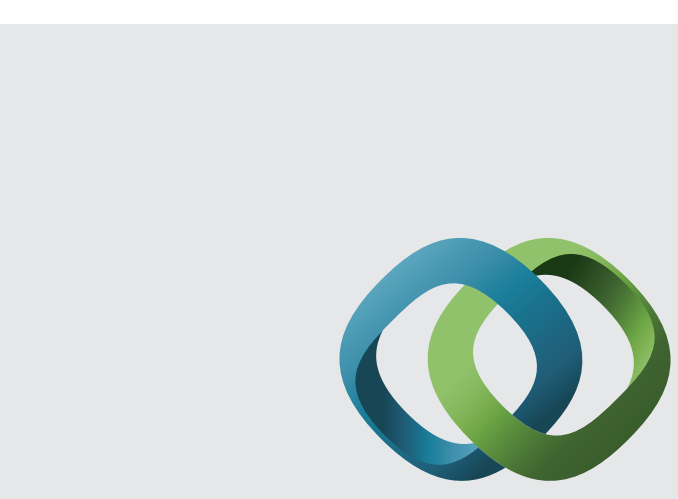

\section{Hindawi}

Submit your manuscripts at

http://www.hindawi.com
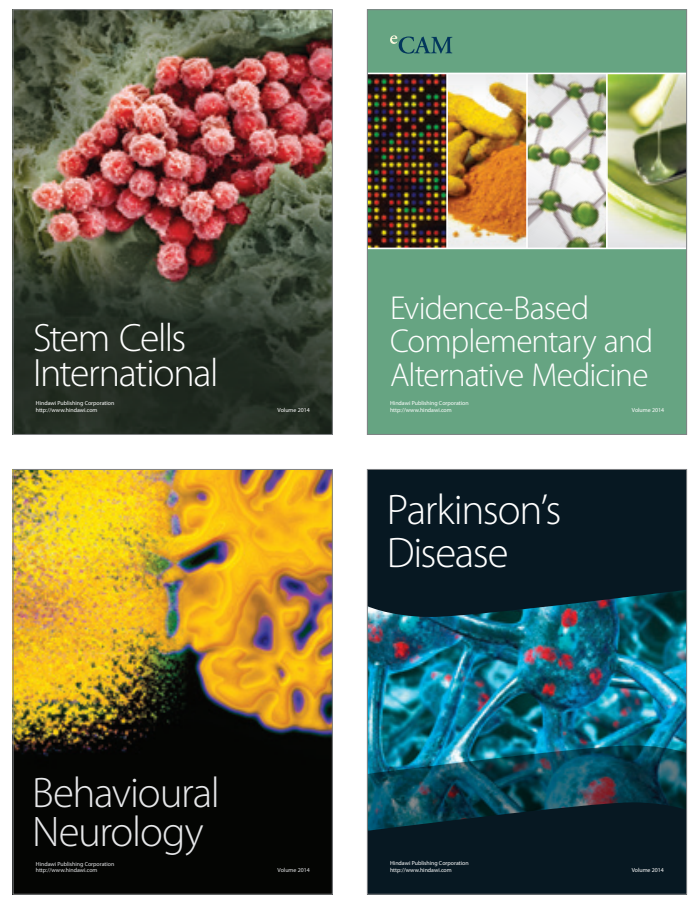
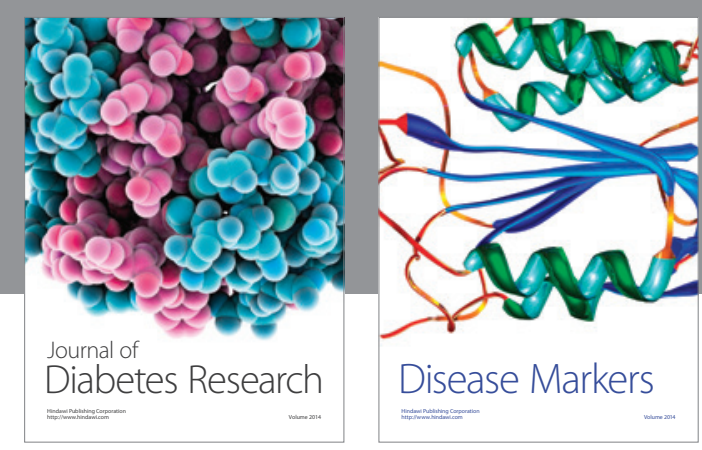

Disease Markers
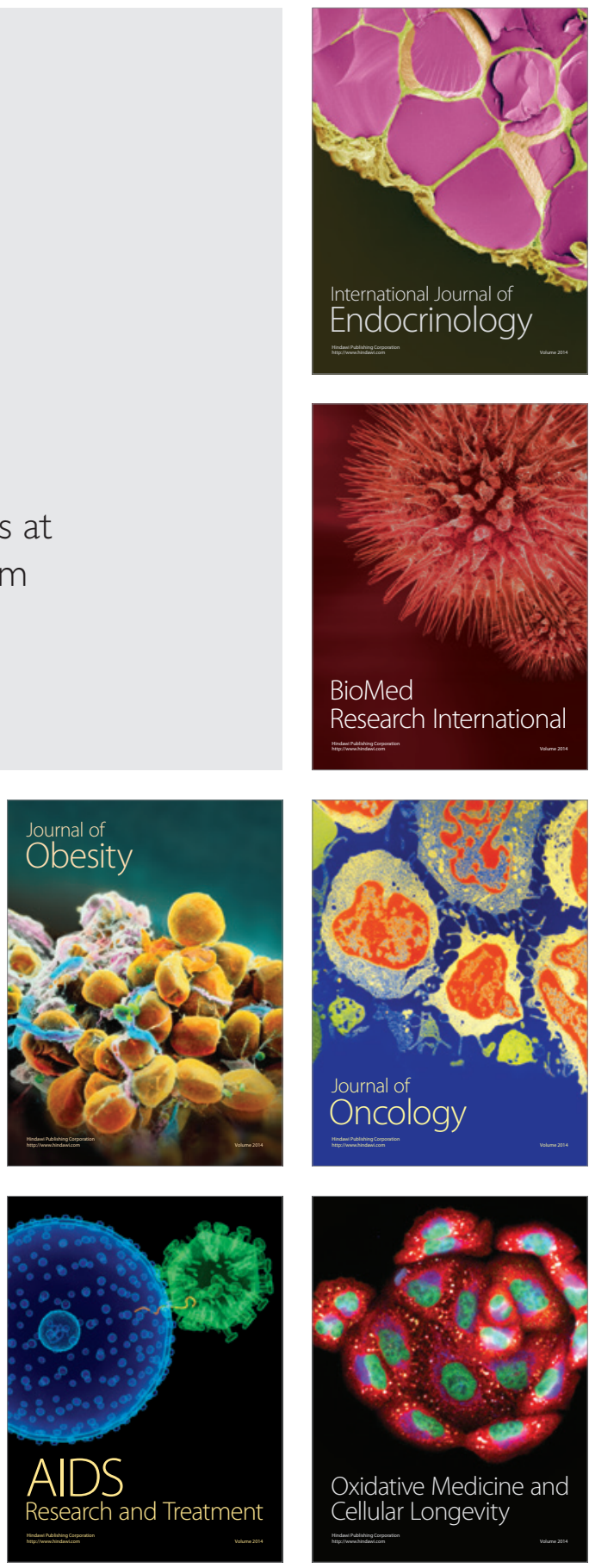